\title{
Fundamental Properties of Linear Ship Steering Dynamic Models
}

\section{Ching-Yaw Tzeng}

Associate Professor, Institute of Maritime Technology, National Taiwan Ocean University, Keelung, Taiwan, R.O.C.

Ju-Fen Chen

Graduate Student, Institute of Maritime Technology, National Taiwan Ocean University, Keelung, Taiwan, R.O.C

Follow this and additional works at: https://jmstt.ntou.edu.tw/journal

Part of the Engineering Commons

\section{Recommended Citation}

Tzeng, Ching-Yaw and Chen, Ju-Fen (2009) "Fundamental Properties of Linear Ship Steering Dynamic Models," Journal of Marine Science and Technology. Vol. 7: Iss. 2, Article 2.

DOI: $10.51400 / 2709-6998.2525$

Available at: https://jmstt.ntou.edu.tw/journal/vol7/iss2/2

This Research Article is brought to you for free and open access by Journal of Marine Science and Technology. It has been accepted for inclusion in Journal of Marine Science and Technology by an authorized editor of Journal of Marine Science and Technology. 


\title{
FUNDAMENTAL PROPERTIES OF LINEAR SHIP STEERING DYNAMIC MODELS
}

\author{
Ching-Yaw Tzeng* and Ju-Fen Chen**
}

Keywords: Nomoto model, Controllability and observability, Identifiability, Model reduction, Overshoot, Bode plot.

\begin{abstract}
This paper is concerned with the fundamental properties associated with the Nomoto models. Specifically, the state space model associated with the first order Nomoto model is both observable and controllable. The state space model associated with the second order Nomoto model is also observable; however, it is controllable only if the effective sway time constant is different from the effective yaw time constant. The zero appearing in the transfer function model is found responsible for the overshoot behaviors, which are typical in the yaw rate for large rudder angle steering. This suggests that a second order Nomoto model is more appropriate if the overshoot feature is to be properly modeled. Both the first and second order Nomoto transfer function models are identifiable, with an ill-conditioning problem associated the latter. This makes the first order Nomoto model very popular in the adaptive autopilot applications. Model reduction for a fourth order transfer function ship model describing the sway-yaw-roll dynamics is conducted to reach the second order Nomoto model describing the sway-yaw dynamics and the first order Nomoto model describing the yaw dynamics itself, and the Bode plots for these models are given to show the changes in system frequency response caused by model simplification. Thus, appropriate model structures can be selected according to the intended frequency range of application to meet the modeling accuracy requirements.
\end{abstract}

\section{INTRODUCTION}

Ship response in waves is typically treated as a six degree-of-freedom rigid body motion in space. Whereas, a three degree-of-freedom plane motion is usually considered adequate for ship maneuvering study [1]. However, for high speed vessels like the container ships, turning motion induced roll is not negligible. Hence a four degree-of-freedom description that in-

Paper Received August 10, 1999. Revised September 17, 1999. Accepted October 25, 1999. Author for Correspondence: Ching-Yaw Tzeng.

*Associate Professor, Institute of Maritime Technology, National Taiwan Ocean University, Keelung, Taiwan, R.O.C.

**Graduate Student, Institute of Maritime Technology, National Taiwan Ocean University, Keelung, Taiwan, R.O.C. cludes surge, sway, yaw and roll modes is needed [2, 3]. Since the hydrodynamics involved in ship steering is highly nonlinear, coupled nonlinear differential equations are needed to fully describe the complicated ship maneuvering dynamics.

A simple transfer function model description is usually preferred when a qualitative prediction capability is all we need from the model. This is the case in a model-based controller design, since the feedback controller itself tolerates certain amount of modeling error and a complicated model might result in a controller too complicated to implement. The popularity of the first order Nomoto model in ship steering autopilot design is due to its simplicity and relative accuracy in describing the course-keeping yaw dynamics, where typically, small rudder angles are involved [4,5]. Extension to large rudder angle yaw dynamics basing upon the Nomoto model has been proposed to better describe the nonlinear behavior of yaw dynamics [6].

Fundamental properties like the controllability and observability of linear systems are known to be important in the design for controllers. The controllability property ensures that all the system states can be driven to desired values with the control input. The observability property ensures that all the system states can be retrieved from the measured output [7]. Specifically, the controllability property itself ensures the state-feedback controllers can be successfully implemented. The controllability and observability properties together ensure the output-feedback controllers can be successfully implemented. The identifiability property ensures that the system model parameters can be uniquely determined from measured input output data, which is crucial to the design of adaptive control systems $[8,9]$.

Generally speaking, state space models are less identifiable than transfer function models. Ship steering state space dynamics models are also known to have identification difficulties due to the cancellation effect [10]. Since most adaptive ship steering autopilot designs are based on transfer function models, our identifibaility study will be concerned with the Nomoto 
transfer function models. However, the controllability and obsevability issues have to be concerned with the state space models. This is because the transfer function models only represent the controllable and observable parts of the system dynamics. Namely, in transforming the state space models into the transfer function models, if there is any uncontrollable or unobservable mode, it will be cancelled out and can not be seen in the transfer function model. Hence, it is the state space counterparts of the Nomoto transfer function models will be used in the study for the controllability and observability properties.

Overshoot behaviors are typical for underdamped oscillatory system. For plane motion-based ship steering dynamics, there is no restoring forces involved. Hence the yaw rate and sway speed will not exhibit oscillatory behaviors. However, for large rudder angle steering, overshoot behaviors in the yaw rate can be observed. It will be shown that this yaw rate overshoot phenomenon is due to coupling effect from the sway mode. A transfer function point of view will be taken to show that the sway coupling effect introduces a zero and a high frequency pole into the system. Specifically, the location of the zero relative to the imaginary axis on the overshoot behavior will be explored and the importance of selecting the appropriate model structure in order to capture key hydrodynamic features, like the overshoot behaviors can be better recognized [11].

Finally, model reduction with respect to a fourth order transfer function models describing the sway-yaw-roll modes of motion will be presented [12]. Simplifications to a second order model and to a first order model describing the sway-yaw and yaw modes respectively are discussed. The Bode plots for these models will be given to illustrate the modeling error incurred due to model simplifications. Based on the frequency response information of the Bode plots, a trade-off between model complexity and model accuracy can be better exercised according to the intended purpose and range of application of the model.

\section{SHIP STEERING DYNAMICS MODEL REDUCTION}

It is well known that coupled nonlinear differential equations are needed to fully represent the complicated ship maneuvering dynamics. However for ship steering autopilots design, a simple model with average predicting capability is usually preferred. Based on the linearized surge-sway-yaw-roll equations of motion, a fourth order transfer function relating the yaw rate to the rudder angle is derived. Further simplifications to the second order Nomoto model and the first order Nomoto model are also described.

Upon linearization with respect to a straight line

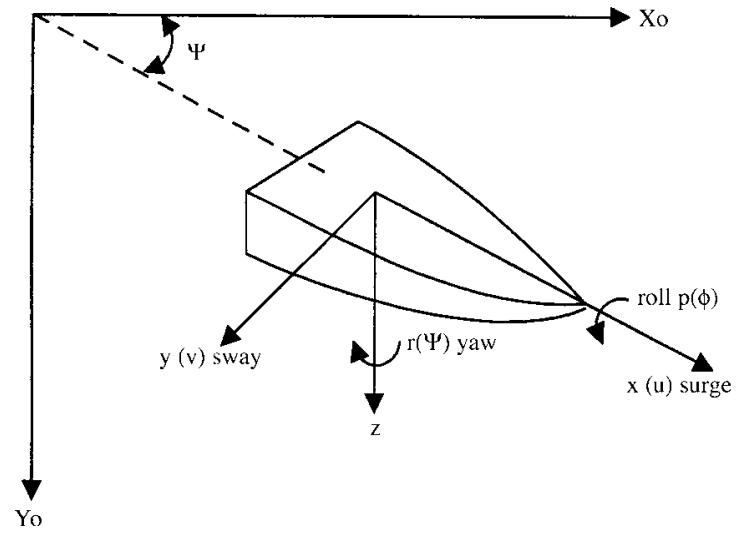

Fig. 1. Sway-yaw-roll motion coordinate system.

motion with a constant forward speed $u_{0}$, the surge equation is decoupled and the following linear coupled sway-yaw-roll equations follow immediately. Referring to Fig. 1, we have

$$
\begin{aligned}
m\left(\dot{v}+u_{0} r\right) & =Y_{V} V+Y_{\dot{V}} \dot{V}+Y_{\varphi} \varphi+Y_{P} P \\
& +Y_{\dot{P}} \dot{P}+Y_{r} r+Y_{\dot{r}} \dot{r}+Y_{\delta} \delta \\
I_{X} \ddot{\varphi}= & K_{P} P+K_{\dot{P}} \dot{P}-m g \overline{G M} \phi+K_{V} V \\
& +K_{\dot{V}} \dot{V}+K_{r} r+K_{\dot{r}} \dot{r}+K_{\delta} \delta \\
I_{Z} \ddot{\psi} & =N_{r} r+N_{\dot{r}} \dot{r}+N_{\phi} \phi+N_{P} P \\
& +N_{\dot{P}} \dot{P}+N_{V} V+N_{\dot{V}} \dot{V}+N_{\delta} \delta
\end{aligned}
$$

where $Y_{V}, Y_{\dot{V}}, \ldots$, indicate the hydrodynamic coefficients; for instance, $Y_{V}$ indicates the derivative of the sway force $Y$ to the sway speed $V$ evaluated at the reference condition; $m$ is the mass of the ship; $I_{X}$ is the moment of inertia about the $\mathrm{x}$-axis; $I_{Z}$ is the moment of inertia about the $z$-axis; $V$ is the sway speed; $u$ is the surge speed; $r$ is the yaw rate; $\Psi$ is the heading angle defined by $\dot{\psi}=r ; p$ is the roll rate; $\phi$ is the roll angle defined by $\dot{\phi}=p$ and $\overline{G M}$ is the metacentric height, which indicates the restoring capability of a ship in rolling motion.

Taking the Laplace transform of Eqs. (1a)-(1c) and rearranging, we have

$$
\begin{aligned}
& a_{1} V=a_{2} \Phi+a_{3} r+a_{4} \delta \\
& b_{1} \Phi=b_{2} V+b_{3} r+b_{4} \delta \\
& c_{1} r=c_{2} V+c_{3} \Phi+c_{4} \delta
\end{aligned}
$$

where 


$$
\begin{aligned}
& a_{1}=\left(m-Y_{\dot{V}}\right) S-Y_{V} \\
& a_{2}=Y_{\dot{P}} S^{2}+Y_{P} S+Y_{\phi} \\
& a_{3}=Y_{\dot{r}} S+Y_{r}+m u_{0} \\
& a_{4}=Y_{\delta} \\
& b_{1}=\left(I_{X}-K_{\dot{P}}\right) S^{2}-K_{P} S+m g \overline{G M} \\
& b_{2}=K_{\dot{V}} S+K_{V} \\
& b_{3}=K_{\dot{r}} S+K_{r} \\
& b_{4}=K_{\delta} \\
& c_{1}=\left(I_{Z}-N_{\dot{r}}\right) S-N_{r} \\
& c_{2}=N_{\dot{V}} S+N_{V} \\
& c_{3}=N_{\dot{P}} S^{2}+N_{P} S+N_{\phi} \\
& c_{4}=N_{\delta}
\end{aligned}
$$

After eliminating the sway speed $V$ and roll angle $\phi$ from Eqs. (2a)-(2c), the following transfer function relating the yaw rate $r$ to the rudder angle $\delta$ can be obtained:

$\frac{r}{\delta}=\frac{a_{1}\left(b_{1} c_{4}+b_{4} c_{3}\right)+a_{2}\left(b_{4} c_{2}-b_{2} c_{4}\right)+a_{4}\left(b_{1} c_{2}+b_{2} c_{3}\right)}{a_{1}\left(b_{1} c_{1}-b_{3} c_{3}\right)-a_{2}\left(b_{2} c_{1}+b_{3} c_{2}\right)-a_{3}\left(b_{1} c_{2}+b_{2} c_{3}\right)}$

It can be easily verified that the numerator of Eq. (4) is third order in S, while the denominator is fourth order in S. Hence, Eq. (4) can be expressed in the following form

$$
\frac{r}{\delta}=\frac{K\left(1+T_{3} S\right)\left(S^{2}+2 \eta \omega_{0} S+\omega_{0}^{2}\right)}{\left(1+T_{1} S\right)\left(1+T_{2} S\right)\left(S^{2}+2 \xi \omega_{n} S+\omega_{n}^{2}\right)}
$$

where the quadratic factors are due to the coupling effect from the roll mode on the yaw rate. The zero (1 $\left.+T_{3} S\right)$ and the pole $\left(1+T_{2} S\right)$ are due to the coupling effect from the sway mode on the yaw dynamics.

If the roll mode is neglected, Eq. (5) can be further reduced to the following form

$$
\frac{r}{\delta}=\frac{K\left(1+T_{3} S\right)}{\left(1+T_{1} S\right)\left(1+T_{2} S\right)}
$$

Eq. (6) is known as the second order Nomoto model, where $K$ is the static yaw rate gain, and $T_{1}, T_{2}$ and $T_{3}$ are time constants. Numerical values of the param- eters in Eq. (6) for a Mariner class vessel are given by $T_{1}$ $=118, T_{2}=7.8, T_{2}=18.5$ and $K=0.185$ [7]. The zero term $\left(1+T_{3} S\right)$ and the high frequency pole term $(1+$ $T_{2} S$ ) are due to the coupling effect from the sway mode.

In practice, because the pole term $\left(1+T_{2} S\right)$ and the zero term $\left(1+T_{3} S\right)$ in Eq. (6) nearly cancel each other, a further simplification on Eq. (6) can be done to give the first order Nomoto model

$$
\frac{r}{\delta}=\frac{K}{(1+T S)}
$$

where

$$
T=T_{1}+T_{2}-T_{3}
$$

in Eq. (8) is called the effective yaw rate time constant. Eq. (8) is obtained by equating the right handside of Eq. (6) to the right handside of Eq. (7) requiring the equality relationship to hold up to first order in $\mathrm{S}$. If $T_{2}=T_{3}$; namely, a perfect cancellation occurs, then the equality relationship is true up to second order in $\mathrm{S}$, then $\mathrm{T}$ is of course, equal to $T_{1}$. For the Mariner class vessel, the value of the effective constant $T$ in Eq. (8) is given by $T$ $=107.3$.

The first order Nomoto model defined by Eq. (7) is widely employed in the ship steering autopilot design. The yaw dynamics is characterized by the parameters $K$ and $T$, which can be easily identified from standard maneuvering tests. In practice, ship steering autopilots are design for heading angle control. Hence, it is the transfer function relating the heading angle $\Psi$ to the rudder angle $\delta$ being needed in the autopilot design. Since the yaw rate $r$ is actually the time derivative of $\Psi$, the required transfer function can be readily obtained by adding an integrator $1 / S$ to the transfer function models defined by Eq. (6) and Eq. (7).

\section{FUNDAMENTAL PROPERTIES OF THE FIRST ORDER NOMOTO MODEL}

The controllability, observability and identifiability properties of the first order Nomoto model-based system will be discussed in this section. The identifiability property will be discussed with respect to the first order Nomoto transfer function model. However, the controllability and observability properties have to be discussed with respect to the state-space model derived from the Nomoto model. This is because the transfer function model always represents the observable and controllable parts of the system dynamics. If there is any unobservable or uncontrollable parts of the dynamics, they are cancelled out before reaching the transfer function model form. Hence, it only makes sense to discuss the controllability and observability 
properties of the state space model, which contains the observable, unobservable, controllable and uncontrollable modes [11].

Eq. (7) can be expressed in time domain as

$$
T \dot{r}+r=K \delta
$$

With the notation

$$
\dot{\psi}=r
$$

where $\Psi$ is the heading of the ship. Eq. (9) can be written as

$$
T \ddot{\psi}+\dot{\psi}=K \delta
$$

Eq. (10) and Eq. (11) can be arranged in the standard state space form

$$
\begin{aligned}
& \dot{x}=A x+B u \\
& y=C x
\end{aligned}
$$

where

$$
\begin{aligned}
& x=\left[\begin{array}{l}
\psi \\
r
\end{array}\right] \\
& u=\delta \\
& y=\Psi
\end{aligned}
$$

and

$$
\begin{aligned}
& A=\left[\begin{array}{cc}
0 & 1 \\
0 & -\frac{1}{T}
\end{array}\right] \\
& B=\left[\begin{array}{c}
0 \\
K / T
\end{array}\right] \\
& C=\left[\begin{array}{ll}
1 & 0
\end{array}\right]
\end{aligned}
$$

According to linear system theory, the system defined by Eqs. (12) is controllable if the following matrix $U$ is of full rank

$$
\begin{aligned}
U & =\left[\begin{array}{ll}
B & A B
\end{array}\right] \\
& =\left[\begin{array}{cc}
0 & K / T \\
K / T & -K / T^{2}
\end{array}\right]
\end{aligned}
$$

and the system is observable if the following matrix $V$ is of full rank

$$
\begin{aligned}
V & =\left[\begin{array}{c}
C \\
C A
\end{array}\right] \\
& =\left[\begin{array}{ll}
1 & 0 \\
0 & 1
\end{array}\right]
\end{aligned}
$$

Straightforward computation indicates that the matrix $U$ and the matrix $V$ defined by Eq. (13) and Eq. (14) respectively are full rank. Hence, the first order Nomoto model-based system is both controllable and observable. Eq. (12b) describes the measurement information. According to Eq. (12h), the measured signal is the heading angle $\Psi$, which is readily available onboard almost all the vessels.

In the above discussion, controllability indicates the system states $(\Psi, r)$ can be controlled to arbitrary value via application of the rudder $\delta$. Observability indicates the system states $(\Psi, r)$ can be obtained via the measured data $\Psi$. Moreover, controllability implies the state-feedback controller will be successful. With the addition of observability implies that the output-feedback controller will be successful.

Identifiability of the first order Nomoto model defined by Eq. (7) implies that the parameters $K$ and $T$ can be uniquely determined from the input rudder angle $\delta$ and the output yaw rate $r$. Since, this is equivalent to fitting a first order model to the measured input-output data, and the gain $K$ and the time constant $T$ are uniquely determined. Consequently, the first order Nomoto model satisfies the identifiability property. Hence, on-line estimation of the model parameters based on the measured rudder and yaw rate information will be possible and adaptive control strategy can be successfully implemented.

\section{FUNDAMENTAL PROPERTIES OF THE SECOND ORDER NOMOTO MODEL}

Similar to previous discussions the identifiability condition will be discussed basing upon the transfer function model defined by Eq. (7), and the controllability and observability conditions will be discussed basing upon the corresponding state space model.

Recall that the second order model Nomoto model defined by Eq. (6) is obtained from Eq. (1a) and Eq. (1c), while neglecting the roll mode. Namely,

$$
\begin{aligned}
& m\left(\dot{v}+u_{0} r\right)=Y_{v} v+Y_{\dot{v}} \dot{v}+Y_{r} r+Y_{\dot{r}} \dot{r}+Y_{\delta} \delta \\
& I_{z} \dot{r}=N_{v} v+N_{\dot{v}} v+N_{r} r+N_{\dot{r}} \dot{r}+N_{\delta} \delta
\end{aligned}
$$

Eqs. (15) can be put in state space format as 


$$
\begin{gathered}
{\left[\begin{array}{ccc}
m-Y_{\dot{v}} & -Y_{\dot{r}} & 0 \\
-N_{\dot{v}} & I_{z}-N_{\dot{r}} & 0 \\
0 & 0 & 1
\end{array}\right]\left[\begin{array}{l}
\dot{v} \\
\dot{r} \\
\dot{\psi}
\end{array}\right]+\left[\begin{array}{ccc}
-Y_{v} & m u_{0}-Y_{r} & 0 \\
-N_{v} & -N_{r} & 0 \\
0 & 1 & 0
\end{array}\right]\left[\begin{array}{c}
v \\
r \\
\psi
\end{array}\right]} \\
=\left[\begin{array}{c}
Y_{\delta} \\
N_{\delta} \\
0
\end{array}\right] \delta
\end{gathered}
$$

Eq. (16) can be further rearranged in the standard state space format as

$$
\begin{aligned}
& \dot{x}=A x+B u \\
& y=C x
\end{aligned}
$$

where

$$
\begin{aligned}
& x=\left[\begin{array}{l}
v \\
r \\
\psi
\end{array}\right] \\
& u=\delta \\
& y=\Psi
\end{aligned}
$$

and

$$
\begin{aligned}
& A=\left[\begin{array}{ccc}
a_{11} & a_{12} & 0 \\
a_{21} & a_{22} & 0 \\
0 & 1 & 0
\end{array}\right] \\
& B=\left[\begin{array}{c}
b_{11} \\
b_{21} \\
0
\end{array}\right] \\
& C=\left[\begin{array}{lll}
0 & 0 & 1
\end{array}\right]
\end{aligned}
$$

The elements in matrix $A$ and $B$ are given by

$$
\begin{aligned}
& a_{11}=\frac{\left(I_{z}-N_{\dot{r}}\right) Y_{v}+Y_{\dot{r}} N_{v}}{\left(m-Y_{\dot{v}}\right)\left(I_{z}-N_{\dot{r}}\right)-N_{\dot{v}} Y_{\dot{r}}} \\
& a_{12}=\frac{\left(I_{z}-N_{\dot{r}}\right)\left(Y_{v}-m u_{0}\right)+Y_{\dot{r}} N_{r}}{\left(m-Y_{\dot{v}}\right)\left(I_{z}-N_{\dot{r}}\right)-N_{\dot{v}} Y_{\dot{r}}} \\
& a_{21}=\frac{\left(m-Y_{\dot{v}}\right) N_{v}+N_{\dot{v}} Y_{v}}{\left(m-Y_{\dot{v}}\right)\left(I_{z}-N_{\dot{r}}\right)-N_{\dot{v}} Y_{\dot{r}}} \\
& a_{22}=\frac{\left(m-Y_{\dot{v}}\right) N_{r}+N_{\dot{v}}\left(Y_{v}-m u_{0}\right)}{\left(m-Y_{\dot{v}}\right)\left(I_{z}-N_{\dot{r}}\right)-N_{\dot{v}} Y_{\dot{r}}}
\end{aligned}
$$

$$
\begin{aligned}
& b_{11}=\frac{\left(I_{z}-N_{\dot{r}}\right) Y_{\delta}+Y_{\dot{r}} N_{\delta}}{\left(m-Y_{\dot{v}}\right)\left(I_{z}-N_{\dot{r}}\right)-N_{\dot{v}} Y_{\dot{r}}} \\
& b_{21}=\frac{\left(m-Y_{\dot{v}}\right) N_{\delta}+N_{\dot{v}} N_{\delta}}{\left(m-Y_{\dot{v}}\right)\left(I_{z}-N_{\dot{r}}\right)-N_{\dot{v}} Y_{\dot{r}}}
\end{aligned}
$$

Controllability of the second order Nomoto modelbased system described Eqs. (17) is satisfied if the following matrix $U$ is of full rank

$$
U=\left[\begin{array}{lll}
B & A B & A^{2} B
\end{array}\right]
$$

$$
=\left[\begin{array}{ccc} 
& a_{11}^{2} b_{11}+a_{11} a_{12} b_{21} \\
b_{11} & a_{11} b_{11}+a_{12} b_{21} & +a_{12} a_{21} b_{11}+a_{12} a_{22} b_{21} \\
& & a_{21} a_{11} b_{11}+a_{21} a_{12} b_{21} \\
b_{21} & a_{21} b_{11}+a_{22} b_{21} & +a_{22} a_{21} b_{11}+a_{22}^{2} b_{21} \\
0 & b_{21} & a_{21} b_{11}+a_{22} b_{21}
\end{array}\right]
$$

After some computation, it can be verified that the matrix $U$ defined by Eq. (18) is of full rank if

$$
a_{12} b_{21}-a_{22} b_{11} \neq b_{11}\left(a_{21} b_{11}-a_{11} b_{21}\right)
$$

Alternatively, the condition defined by Eq. (19) can be verified by requiring the columns in Eq. (18) not proportional to each other and the rows in Eq. (18) not proportional each other. Controllability condition of the system defined by Eqs. (17) implies that the states $\Psi, r$ and $v$ can be driven independently to arbitrary values via the rudder angle $\delta$.

Implication of the controllability condition will be further explored. Recall that by eliminating the sway velocity $v$ in Eq. (15a) and Eq. (15b) leads to the second order Nomoto model defined by Eq. (6). Similarly by eliminating the yaw rate $r$ in Eq. (15a) and Eq. (15b) leads to the sway to rudder transfer function

$$
\frac{v}{\delta}=\frac{K_{v}\left(1+T_{v} S\right)}{\left(1+T_{1} S\right)\left(1+T_{2} S\right)}
$$

where $K_{v}$ is the static sway gain coefficient and $T_{v}$ is the sway time constant. It is to be noted that the poles of the sway-rudder model defined by Eq. (20) are exactly the same as those in the yaw-rudder model defined by Eq. (6).

From Eq. (6) and Eq. (20), it follows that

$$
\frac{v}{r}=\frac{K_{v}\left(1+T_{v} S\right)}{K(1+T S)}
$$

Eq. (21) shows that, if $T_{v}=T$, then $v$ is proportional to $r$. Namely, $v$ is dependent on $r$, since $K_{v} / K$ is a 


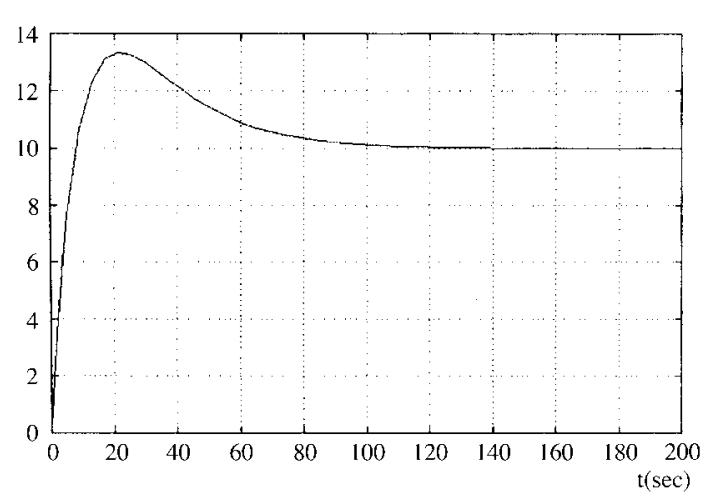

Fig. 2. Unit step response, $\frac{10(1+40 S)}{(1+10 S)(1+20 S)}$.

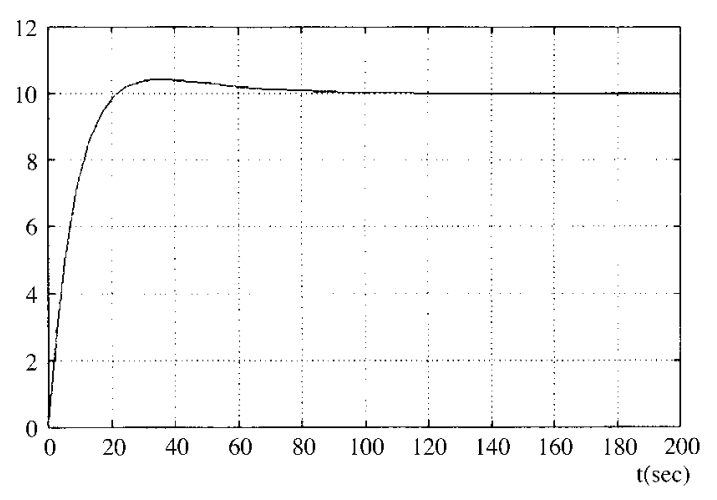

Fig. 3. Unit step response, $\frac{10(1+25 S)}{(1+10 S)(1+20 S)}$.

constant. However, we recall that for the second order Nomoto model-based system defi]Öd by Eqs. (17). If the system is controllable, then the state variables $\Psi, r$, $v$ have to be able to move independently via application of the rudder $\delta$. It is thus inferred that for the system to be controllable, $T_{v}$ must be different from $T$. Namely, $T_{v}$ $\neq T$ is equivalent to the condition given in Eq. (19) that ensures the controllability of the system.

Observability condition of the system defined by Eqs. (17) is satisfied if the following matrix $V$ is of full rank

$$
\begin{aligned}
V & =\left[\begin{array}{c}
C \\
C A \\
C A^{2}
\end{array}\right] \\
& =\left[\begin{array}{ccc}
0 & 0 & 1 \\
0 & 1 & 0 \\
a_{21} & a_{22} & 0
\end{array}\right]
\end{aligned}
$$

It can be easily verified that Eq. (22) is of full rank for any ship. Hence, the second order Nomoto model-

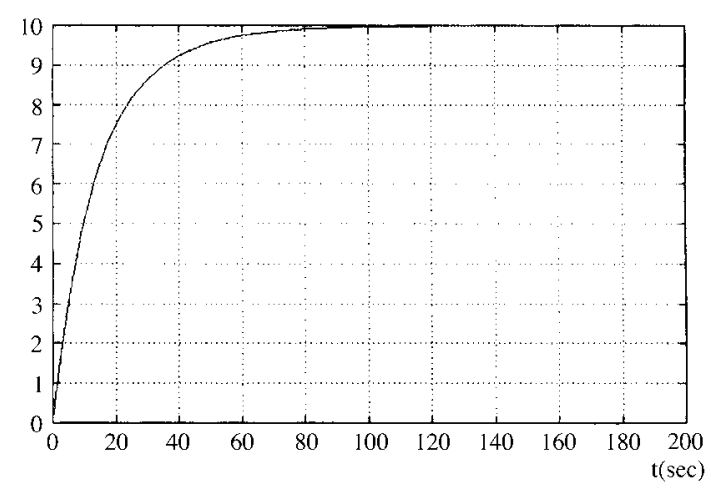

Fig. 4. Unit step response, $\frac{10(1+15 S)}{(1+10 S)(1+20 S)}$.

based state space system is observable. This implies that all the states $(\Psi, r, v)$ can be reconstructed from the measured heading angle $\Psi$.

Identifiability of the second order Nomoto model defined by Eq. (6) implies that the parameters $K, T_{1}, T_{2}$ and $T_{3}$ appearing in Eq. (6) can be uniquely determined from the input $\delta$ and output $r$ measurements. It is clear that if $T_{2}=T_{3}$, then it is impossible to identify all the four parameters $K, T_{1}, T_{2}$ and $T_{3}$. Since the zero term (1 $\left.+T_{3} S\right)$ will cancel out the pole term $\left(1+T_{2} S\right)$. It is then inferred that the system defined by Eq. (6) is identifiable if $T_{2} \neq T_{3}$.

In practice, the value of $T_{2}$ is nearly equal to the value of $T_{3}$ and we have a near cancellation situation. This will result in an ill-conditioning problem when trying to identify the values of $T_{2}$ and $T_{3}$ appearing in the second order Nomoto model defined by Eq. (6). Due to this ill-conditioning property, the first order Nomoto model defined by Eq. (7) is preferred to Eq. (6) in the design for an adaptive autopilot, where reliable on-line estimation of the model parameters is needed.

\section{SYSTEM OVERSHOOT AND ZERO LOCATION}

In this section, the effect of the zero term appearing in the second order Nomoto model defined by Eq. (6) will be studied. Unit step response will be given to illustrate the relation between the zero location and the existence of overshoot behaviors. Unit step responses for the second order Nomoto model defined by Eq. (6) with the following numerical data will be presented. Specifically, $K=10, T_{1}=10, T_{2}=20$. Four values will be assigned to $T_{3}$ to represent different locations of the zero. Specifically, Fig. 2 corresponds to the unit step response for $T_{3}=40$, Fig. 3 corresponds to $T_{3}=25$, Fig. 4 corresponds to $T_{3}=15$ and Fig. 5 corresponds to $T_{3}=5$ respectively. The overshoots are observed in Fig. 2 and Fig. 3 but do not appear in Fig. 4 
and Fig. 5. Moreover, the overshoot is larger in Fig. 2 than that in Fig. 3. By examing the position of the zero relative to the pole, it is found that the closer the zero is located near the imaginary axis, the larger the overshoot is. Moreover, for the overshoot to exist, the zero must be located to the right of the poles.

In practice the overshoot in the yaw rate $r$ is observed in large rudder angle turning maneuver. Thus, it is inferred that the second order Nomoto model has to be employed, if the overshoot phenomenon is to be captured. Since the first order Nomoto model can never exhibit an overshoot phenomenon, it is indeed only suitable for the description of small rudder angle yaw dynamics, where overshoot in the yaw rate $r$ will not appear.

Moreover, for the steering dynamics, there is no hydrodynamic restoring force involved. Hence the yaw rate $r$ will not have an oscillatory behavior and the ship steering dynamics will always act like an over-damped system. Consequently, the poles are always of real values and this further justifies that the second order Nomoto model that has two real poles is indeed appropriate in describing the plane motion-based ship maneuvering dynamics. Recall that when reducing the second order Nomoto model defined by Eq. (6) to the first order Nomoto model defined by Eq. (7) actually neglects the sway coupling effect on the yaw mode. The zero and the high frequency pole are then neglected. Thus it can be inferred that the overshoot in the yaw rate $r$ is actually caused by the sway coupling effect, and the tighter the maneuver is, the larger the sway coupling effect is. From the above discussions it is concluded that the first order Nomoto model is suitable for describing the small rudder angle yaw dynamics, and needs only two parameters to characterize the system behavior. This makes it relatively easy to identify the model from experiment data. The second order Nomoto model has better capability in capturing the overshoot behavior. However, near cancellation of one of the poles and zero makes it an ill-conditioning problem in identifying the model parameters from experiment data makes it less attractive for an adaptive autopilot application.

In this paper, an alternative approach is suggested for an adaptive autopilot implementation basing upon the second order Nomoto model. Since the zero of the transfer function helps better describing the yaw dynamic overshoot behavior, its structure is retained and the parameter is fixed at a value determined off-line from input-output experiment data. The model described by Eq. (6) is thus unchanged, except that the parameter $T_{3}$ is fixed. Hence, only the parameter $K, T_{1}$ and $T_{2}$ need to be identified on-line. This strategy preserves the feature of the second order Nomoto model without introducing the ill-conditioning problem in iden-

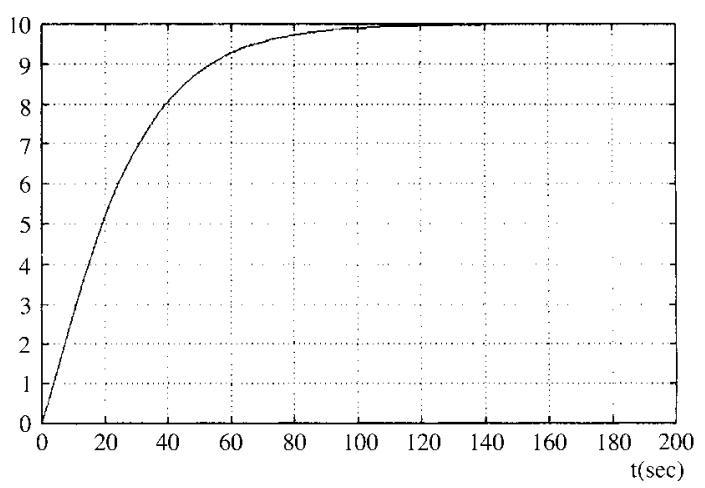

Fig. 5. Unit step response, $\frac{10(1+5 S)}{(1+10 S)(1+20 S)}$.

tifying the model parameters makes it more attractive than the conventional implementation of an adaptive autopilot based on the first order Nomoto model defined by Eq. (7).

\section{MODEL SIMPLIFICATION AND BODE PLOTS}

A fourth order linear state space model representing the sway-yaw-roll modes of motion given in Ref. [12] will be used as the nominal model in constructing the corresponding yaw to rudder transfer function. Further simplification to the second order Nomoto model and the first order Nomoto model will also be presented. The nominal state space model is given by

$$
\begin{aligned}
& \dot{x}=A x+B u \\
& y=C x
\end{aligned}
$$

where

$$
\begin{aligned}
& x=\left[\begin{array}{l}
\phi \\
p \\
v \\
r
\end{array}\right] \\
& u=\delta \\
& y=r
\end{aligned}
$$

and

$$
A=\left[\begin{array}{cccc}
0 & 1 & 0 & 0 \\
-0.059 & -0.07 & -0.00594 & 0 \\
-0.0756 & 0 & -0.04 & -1.933 \\
0.001134 & 0 & -0.00011-0.0813
\end{array}\right]
$$




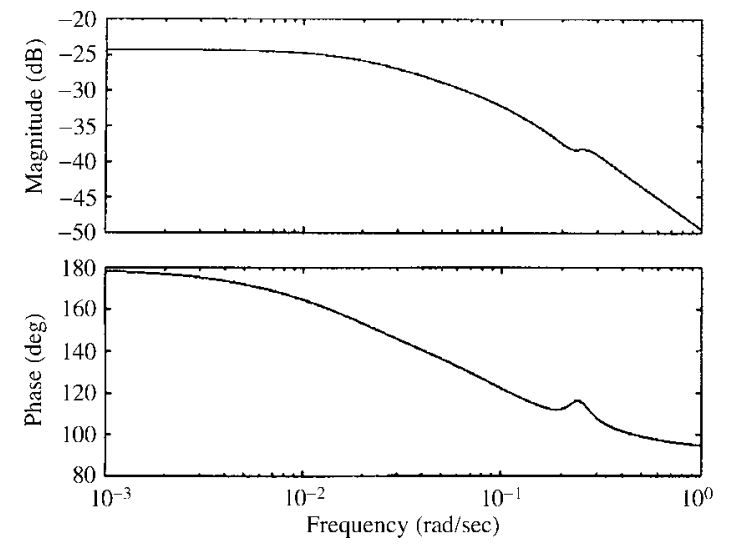

Fig. 6. Bode diagrams, fourth order model.

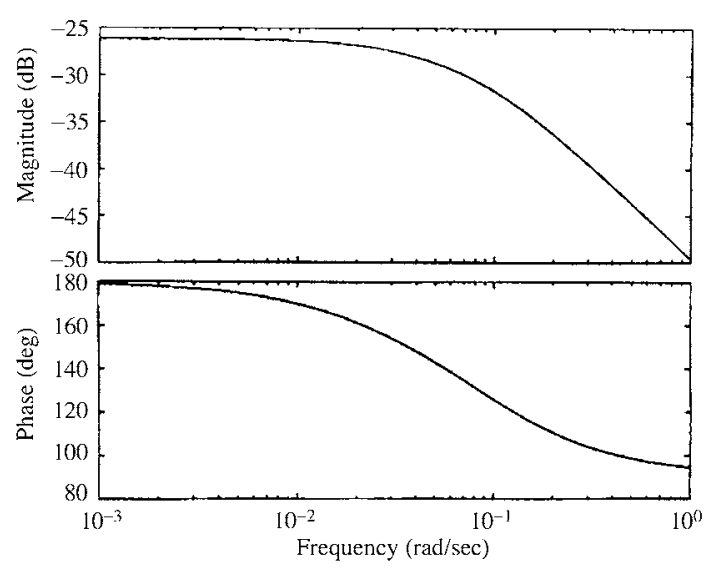

Fig. 7. Bode diagrams, second order model.

$$
\begin{aligned}
& B=\left[\begin{array}{c}
0 \\
0.00821 \\
0.1559 \\
-0.0033
\end{array}\right] \\
& C=\left[\begin{array}{llll}
0 & 0 & 0 & 1
\end{array}\right]
\end{aligned}
$$

Using the MATLAB, the transfer function from input rudder $\delta$ to the output yaw rate $r$ is obtained as

$$
\frac{r}{\delta}=\frac{-0.0033 S^{3}-0.00038 S^{2}-0.000195 S-0.000079}{S^{4}+0.1913 S^{3}+0.075 S^{2}+0.0692 S+0.00013}
$$

The numerator and denominator of Eq. (24) are of third order and fourth order in $S$ respectively, and this agrees with that of Eq. (4). It is to be noted that Eq. (24) represents the yaw dynamics with inclusion of the roll and sway coupling effects.

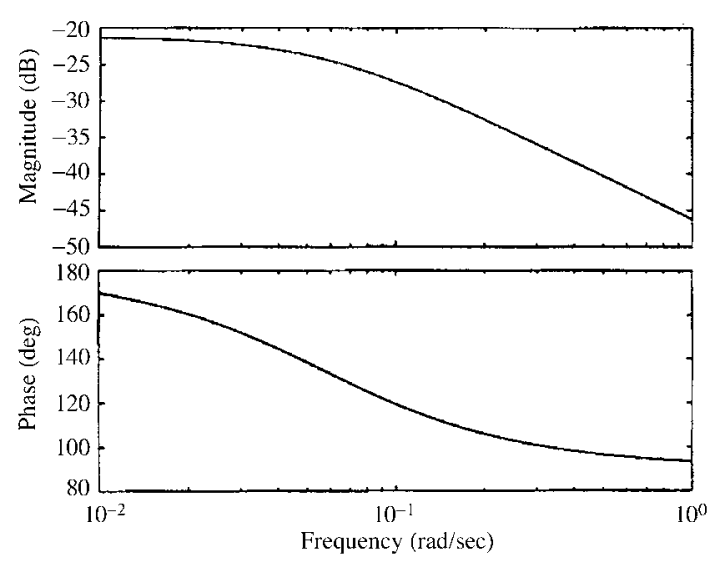

Fig. 8. Bode diagrams, first order model.

By neglecting the roll mode in Eq. (23), the following yaw rate to rudder transfer function that includes the sway coupling effect can be obtained

$$
\frac{r}{\delta}=\frac{-0.0033 S-0.00015}{S^{2}+0.1213 S+0.00304}
$$

Eq. (25) corresponds to the second order Nomoto model defined by Eq. (6) Further neglecting the sway coupling effect results in the model that has yaw mode itself only

$$
\frac{r}{\delta}=\frac{-0.049}{1+17.78 S}
$$

Eq. (26) corresponds to the first order Nomoto model defined by Eq. (7) Bode plots that represent the frequency domain yaw responses of the models defined by Eqs. (24)-(26) are given in Figs. 6-8 respectively. Each figure has a magnitude plot and a phase plot. Specifically, Fig. 6 corresponds to the fourth order model, Fig. 7 corresponds to the second order model and Fig. 8 corresponds to the first order model. The magnitude plot and phase plot of the second order Nomoto model are about the same as those of the fourth order model, expect that there are humps associated with the fourth order model. The difference between the second order Nomoto model and the first order Nomoto model is somewhat larger. Specifically, there is a $5 \mathrm{db}$ magnitude difference and a $20 \mathrm{deg}$ phase difference for the frequency range between $10^{-2} \mathrm{rad} / \mathrm{sec}$ to $10^{-1} \mathrm{rad} / \mathrm{sec}$.

Based on the results of Figs. 6-8, it is clear that the effect of model simplification from the fourth order model to the second order Nomoto model is not significant; namely, the coupling effect of the roll mode on the yaw motion is negligible. However, the effect of model reduction from the second order Nomoto model to the first order Nomoto model is more significant; namely, the coupling effect of the sway mode on the 


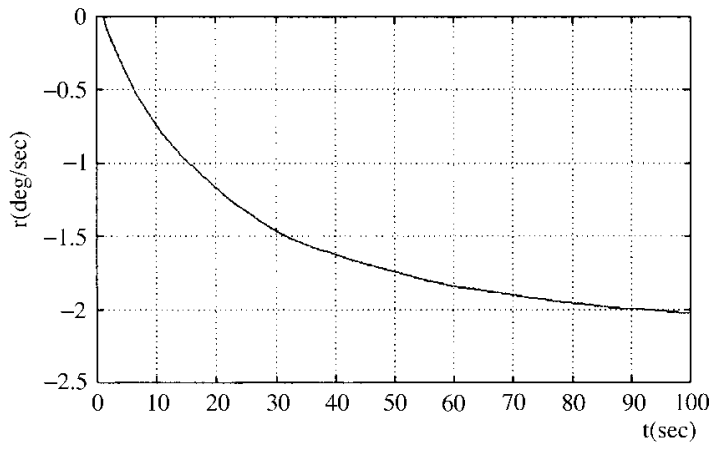

Fig. 9. Yaw rate response due to rudder, fourth order model.

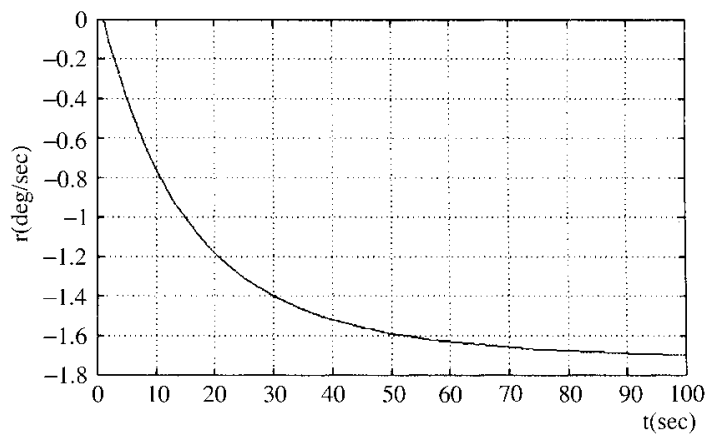

Fig. 10. Yaw rate response due to rudder, second order model.

yaw motion is not negligible. Hence for the ship model reported in Ref. [12], it seems reasonable to use the second order Nomoto model defined by Eq. (25) to represent the behavior of the fourth order model represented by Eq. (24) in an autopilot design.

Step input response of the ship models described by Eqs. (24)-(26) is also given to exhibit their time domain behaviors. Specifically, Figs. 9-11 correspond to the yaw rate response, due to $35 \mathrm{deg}$ rudder input, of a fourth order, second order and first order model respectively.

With the frequency response information of the Bode plots for models with different level of complexity, modeling errors caused by model reduction can be better assessed. The yaw rate response in the time domain provides a convenient way of assessing the behavior of each model. It is well known that a more complicated model tends to better describe the system behavior in a wider frequency range. However, the intended frequency range of a dynamic system is usually limited a finite interval, which is usually roughly known in advance. This information is helpful in choosing the appropriate model that achieves reasonable accuracy in the frequency range of interest.

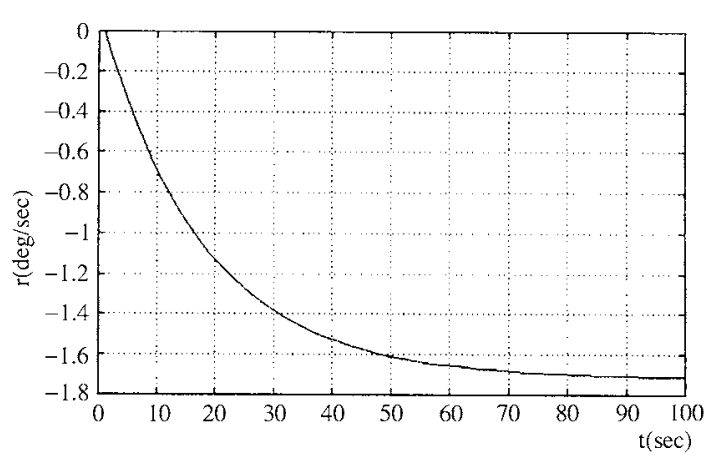

Fig. 11. Yaw rate response due to rudder, first order model.

\section{CONCLUSIONS}

The first order Nomoto model being very popular in the design for ship steering autopilots is not without reason. Its simplicity and reasonable accuracy in describing small rudder angle yaw dynamics make it attractive. Moreover, the relative easiness in identifying the model parameters makes it suitable for adaptive autopilot application, where on-line estimation of the model parameters is important. The second order Nomoto model includes the coupling effect from the sway to the yaw mode. This introduces a zero and a high frequency pole into the transfer function. The zero structure contributes to the overshoot phenomenon, which can be seen in the yaw rate for large rudder angle maneuvers. However, the ill-conditioning problem associated with the second order Nomoto model in the identification of the model parameters from input-output data seems to outweigh the improvement gained in its modeling capability. An approach that retains the zero structure while avoiding the ill-conditioning problem during on-line estimation has been proposed.

The first order Nomoto model's state space counterpart is found to be both controllable and observable; hence, both the state feedback and output feedback controllers can be successfully implemented. The second order Nomoto model's state space counterpart is also observable; however, the system is not controllable, if the effective sway time constant $T_{v}$ is equal to the effective yaw rate time constant $T$. Bode plots of models with different level of complexity are useful in characterizing the relative modeling errors incurred due to model reduction on the frequency domain. With the help of Bode plots, justification of using a simple model can be done within the intended frequency range of application to ensure that the frequency response of the simple model reasonably approximates that of the complicated ones. 


\section{REFERENCES}

1. Lewis, E.V., Editor, "Principles of Naval Architecture, Vol. III Motion in Waves and Controllability," Society of Naval Architects and Marine Engineers, New Jersey (1989).

2. Son, K.H. and Nomoto K., "On the Coupled Motion of Steering and Rolling of High Speed Container Ship", Journal of Society of Naval Architects, Japan, Vol. 150, pp. 232-244 (1981).

3. Oltmann, P., "Roll-An often Neglected Element of Maneuvering," International Conference on marine Simulation and Maneuverability, New Foundland, Canada (1993).

4. Nomoto, K., Taguchi, K., Honda, K. and Hirano, S., "On the Steering Quality of Ships," International Shipbuilding Progress, Vol. 4, pp. 354-370 (1957).

5. van Amerongen, J., "Adaptive Steering of Ships - A Model Reference Approach to Improved Maneuvering and Economical Course-Keeping," Ph.D. Thesis, Delft University of Technology, The Netherlands (1982).

6. Norrbin, N.H., "On the Design and Analysis of the Zig-Zag Test on Base of Quasilinear Frequency Response,” Technical Report No. B140-3, The Sweden State Shipbuilding Experimental Tank (SSPA), Gothenburg, Sweden (1963).

7. Fossen, T.I., "Guidance and Control of Ocean Vehicles," John Wiley and Sons, NY (1994).

8. Astrom, K.J. and Kallstrom, C.G., "Identification of Ship Steering Dynamics," Automatica, Vol. 12, pp. 9-22 (1976).

9. Holzhuter, T. and Strauch, H., "A Commercial Adaptive Autopilot for Ships : Design and Operational Experiences," IFAC 10th Triennial World Conferences, pp. 233-237, Munich, Germany (1987).

10. Hwang, W.Y., "Cancellation Effect and Parameter Identifiability of Ship Steering Dynamics," International Shipbuilding Progress, Vol. 26, No. 332, pp. 90-120 (1982).

11. Goodwin, G.C., Graebe S.E. and Salgado, M.E., "Principles of Control System Design," Department of Electrical and Computer Engineering, University of Newcastle, Callaghan, Australia (1997).

12. Zhou, W.W., Cherchas, D.B. and Calisal, S., "Identification of Rudder-Yaw and Rudder-Roll Steering Models by Using Recursive Prediction Error Techniques", Optimal Control Application and Methods, Vol. 15, pp. 101-114 (1994).

\section{線性船舶操縱模式特性分析}

$$
\text { 曾 慶 耀 }
$$

海洋大學航運技術研究所副教授

$$
\text { 陳如芬 }
$$

海洋大學航運技術研究所研究生

摘 要

船舶操縱運動包含複雜之流力作用現象, 因此 必須使用高階之非線性微分方程式方能完整描述其特 性。然而就船舶自航器設計而言, 多以較簡單之線性 模式, 如一階Nomoto模式爲設計依據。該模式具有 架構簡單, 且足以正確描述航向保持之小舵角船舶平 擺動態之特性。經分析知, 一階Nomoto轉移函數具 有可鑑定性, 而二階之Nomoto轉移函數亦爲可鑑 定, 但其零點與極點通常接近對消之狀況, 因此實際 計算時, 會有數值不穞定現象。一階Nomoto模式所 對應之狀態空間模式則具有可控制性及可觀測性。而 二階之Nomoto模式對應之狀態空間模式爲可觀測, 但必須在横移時間常數不等於平擺時間常數時, 方爲 可控制。二階Nomoto轉移函數模式之零點及高頻之 極點, 乃源自横移對平擺之耦合效應, 且該零點將造 成系統狀態之超射現象。因此對於在大舵角操縱時, 平擺角速率之超射現象, 須使用二階之Nomoto模式 方足以描述之。另亦就一包含横移、平擺及横搖之狀 態空間方程式, 求出對應之四階轉移函數, 並簡化成 包含横移、平擺之二階轉移函數及僅包含平擺之一階 轉移函數。經由Bode頻率響應圖, 可看出不同複雜 程度模式造成之響應特性差異, 該資料可提供使用者 就其需求之模式需求精確度評估, 進而選取簡單卻不 失眞確度之模式。 